\title{
Relationship Between Drug Resistance and Death in HIV-Infected Patients Receiving Antiretroviral Therapy - 7 PLADs, China, 2010-2019
}

\author{
Tianhao Zhang'; Lingjie Liao'; Yiming Shao'; Yi Feng'; Yuhua Ruan ${ }^{1, * *}$; Hui Xing ${ }^{1, *}$
}

\section{Summary \\ What is already known about this topic? \\ With increasing coverage of antiretroviral therapy (ART) for HIV-infected patients, more and more attention has been paid to the impact of HIV drug resistance on death in those patients in China. \\ What is added by this report? \\ Among HIV-infected patients receiving ART, the risk of death is higher in patients with HIV drug resistance [adjusted odds ratio $(\mathrm{AOR})=4.25,95 \%$ confidence interval (CI): $2.10-8.62]$, with viral load $\geq 1,000$ copies $/ \mathrm{mL}$ but drug resistance untested $(\mathrm{AOR}=4.65$, 95\% CI: 1.74-12.39), and with neither viral load nor drug resistance being tested ( $\mathrm{AOR}=17.52$, 95\% CI: 8.73-35.19) when compared with drug-sensitive patients. \\ What are the implications for public health practice? \\ It is important to strengthen drug resistance monitoring and prevention in HIV-infected patients. While performing ART for HIV-infected patients, viral load testing and drug resistance testing should be carried out routinely and promptly.}

To reduce the mortality rate of $\mathrm{HIV}$-infected patients and improve their quality of life, China has launched the "Four Frees and One Care" policy (Free treatment, free voluntary counseling and testing, free prevention of mother to child transmission, free schooling for AIDS orphans, and one "Care": provision of social assistance for HIV/AIDS patients) to provide lifelong free antiretroviral therapy (ART) for HIV-infected patients who meet the national treatment criteria since 2003 (1). In response to the Joint United Nations Programme on HIV/AIDS's (UNAIDS) “Treatment 2.0" strategy, China has implemented a number of targeted strategies to expand the ART coverage in HIV-infected patients since 2010 (2). By the end of 2018, a total of $83.4 \%$
(748,499/861,042) of HIV-infected patients have received ART (3). However, HIV drug resistance (HIVDR) inevitably emerged along with the scale-up of ART, and the drug resistance pattern varied a lot in different regions of China (4). This study analyzed data in the national HIV/AIDS Comprehensive Response Information Management System (CRIMS) to investigate the relationship between HIVDR and death in HIV-infected patients receiving ART in seven provincial-level administrative divisions (PLADs) of China. The main finding is that the risk of death was higher in patients with HIV drug resistance or untested resistance compared with drug-sensitive patients. This helps provide a valuable reference for optimizing ART regimens and patient follow-up management in practice.

This study selected seven PLADs that reported and followed-up a large number of HIV/AIDS patients and considered geographical location (eastern and western China) and economic status. Data was collected for patients who received ART, died from all-causes during 2010-2019, and had adequate blood samples that were collected in twelve months prior to death and after ART initiation date. These samples could satisfy the volume requirements for performing viral load and drug resistance tests and were thus selected as death cases. One or two controls were selected for each case from surviving patients who received treatment at the same ART clinic as the case and was registered right before or after the case. The control should have blood samples collected in six months prior to or after the death cases' blood samples. The eligible cases or controls must be people aged 18 years or over when ART initiated, began their ART treatment in 2010 or later, and received ART for more than 6 months.

The data was derived from the CRIMS whose information was collected by local CDCs or HIV hospitals with questionnaires or medical records. Main variables were survival status, gender, age, ethnicity, marital status, education level, occupation, residence, 
HIV transmission route, ART initiation date, clinic stage according to World Health Organization (WHO) definition, CD4 cell count at the beginning of ART, primary ART regimen, latest ART regimen, ART adherence, latest viral load test result, and latest drug resistance test result.

"HIVDR result" was defined as the main independent variable to analyze the relationship between HIVDR and death (by combining "Latest Viral Load test result" and "Latest drug resistance test result," as shown in Table 1). Due to differences of HIVDR prevalence, quality of medical care services, and sample sizes in regions, multivariate logistic regression analysis was performed for each region, and the adjusted odds ratio (AOR) was obtained. Then, the AORs in different regions were merged by metaanalysis to represent the overall relationship between HIVDR and death in those HIV-infected patients receiving ART. Logistic regression analysis was performed by SAS (version 9.4, SAS Institute, Cary, NC, USA). Meta-analysis was conducted in RevMan (version 5.4, Cochrane Collaboration, Oxford) and NCSS 2004 (Kaysville, UT, USA).

A total of 19,235 HIV-infected patients were enrolled from 7 PLADs (Table 2), with 5,719 in the case group (deaths) and 13,516 in the control group (survived). The proportions of latest viral load untested were $40.0 \%$ in the case group and $17.2 \%$ in the control group, while $65.6 \%$ of case group and $60.8 \%$ of control group were latest drug resistance untested. For the HIVDR result, $25.5 \%$ of case group and $44.2 \%$ of control group were deemed as drug sensitive, and $5.0 \%$ of case group and $2.4 \%$ of control group were drug resistant. The proportions of patients with viral load $\geq 1,000$ copies $/ \mathrm{mL}$ but drug resistance untested were $8.6 \%$ in the case group and $2.7 \%$ in the control group. The proportions of patients who were neither viral load nor drug resistance tested were $28.7 \%$ in the case group and $2.9 \%$ in the control group, respectively.

The multivariate logistic regression analysis indicated that the correlation between the HIVDR result and death was statistically significant $(P<0.05)$ in Anhui, Sichuan, Jiangsu, and Hunan but not in Guangdong $(P>0.05)$. No data was shown on the latest compound variable of viral load and drug resistance in Chongqing and Guangxi. After merging AORs of the HIVDR result with deaths in Anhui, Sichuan, Jiangsu, Guangdong, and Hunan, the result suggested that compared with drug-sensitive patients, the risk of death among patients with drug resistance $[\mathrm{AOR}=4.25$, $95 \%$ confidence interval (CI): 2.10-8.62], with viral load $\geq 1,000$ copies $/ \mathrm{mL}$ but drug resistance untested (AOR=4.65, 95\% CI: 1.74-12.39), and with neither viral load nor drug resistance being tested (AOR $=17.52$, 95\% CI: 8.73-35.19) were statistically significantly higher (Table 3).

\section{DISCUSSION}

A cross-control survey in seven PLADs in China was conducted to investigate the relationship between HIVDR and death in HIV-infected patients receiving ART. A total of 19,235 participants were included in the analysis. Compared with drug-sensitive patients, the risk of death is higher in patients with HIV drug resistance or untested resistance.

In this large sample study, case-control analysis of patients in seven PLADs showed that HIVDR is significantly associated with death in HIV-infected patients receiving ART. Compared with drug-sensitive HIV-infected patients, the risk of death is 3.25 times

TABLE 1. Operational definition and category of HIVDR result.

\begin{tabular}{lll}
\hline \multicolumn{2}{c}{ Primary results of viral load and drug resistance test } & \multicolumn{1}{c}{ Redefined HIVDR categories } \\
\cline { 1 - 2 } \multicolumn{1}{l}{ Latest viral load test result (copies $/ \mathrm{mL})$} & Latest drug resistance test result & \\
\hline$<1,000$ & Sensitivity or not be tested & Drug sensitive \\
$\geq 1,000$ & Sensitivity & Drug sensitive \\
Not be tested & Sensitivity & Drug sensitive \\
$<1,000$ & Resistance & Drug resistant \\
$\geq 1,000$ & Resistance & Drug resistant \\
Not be tested & Resistance & Drug resistant \\
$\geq 1,000$ & Not be tested & Viral load $\geq 1,000$ copies/mL but drug resistance untested \\
Not be tested & Not be tested & Neither viral load nor drug resistance being tested \\
\hline
\end{tabular}

Abbreviation: HIVDR=HIV drug resistance. 


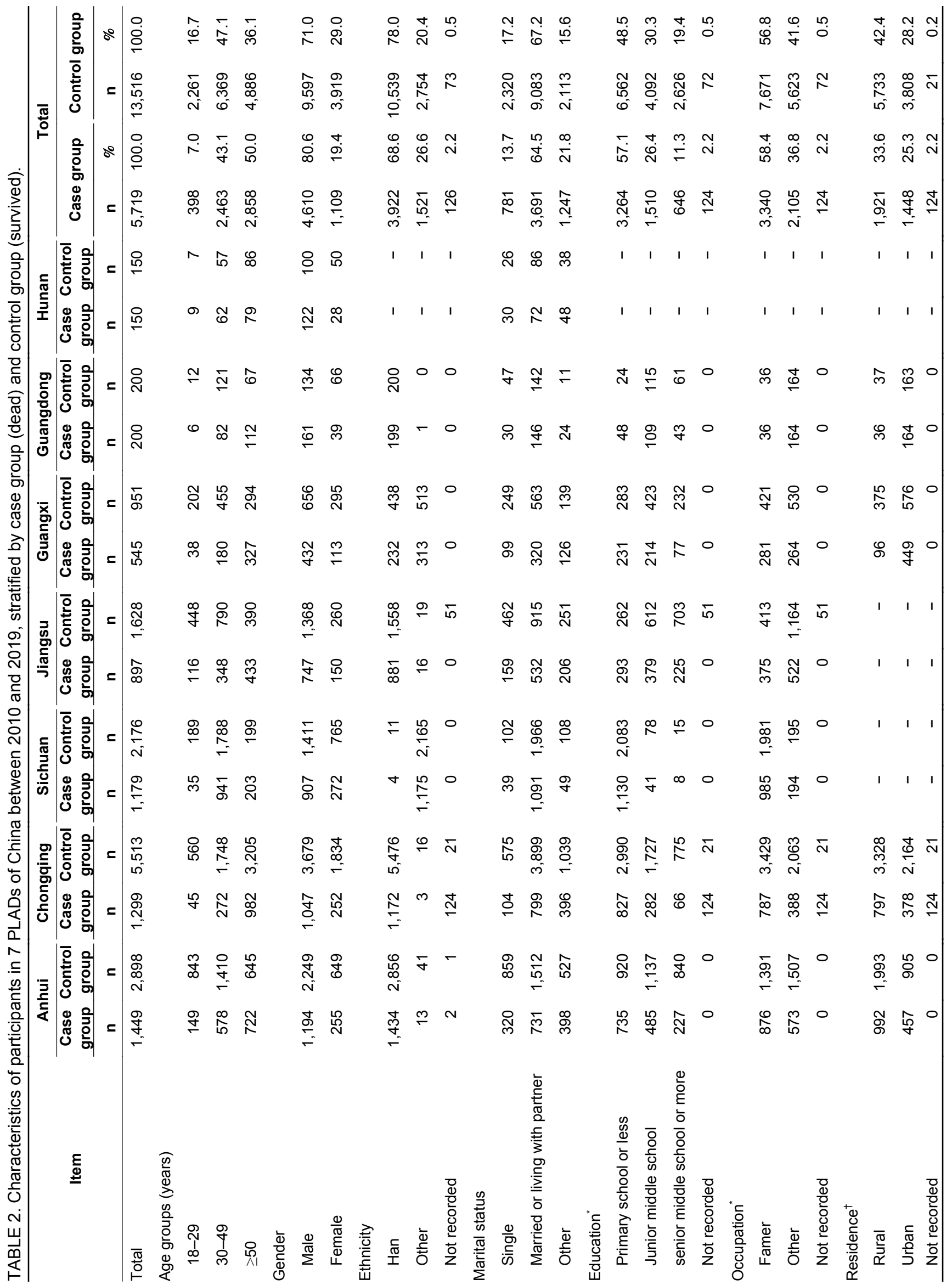




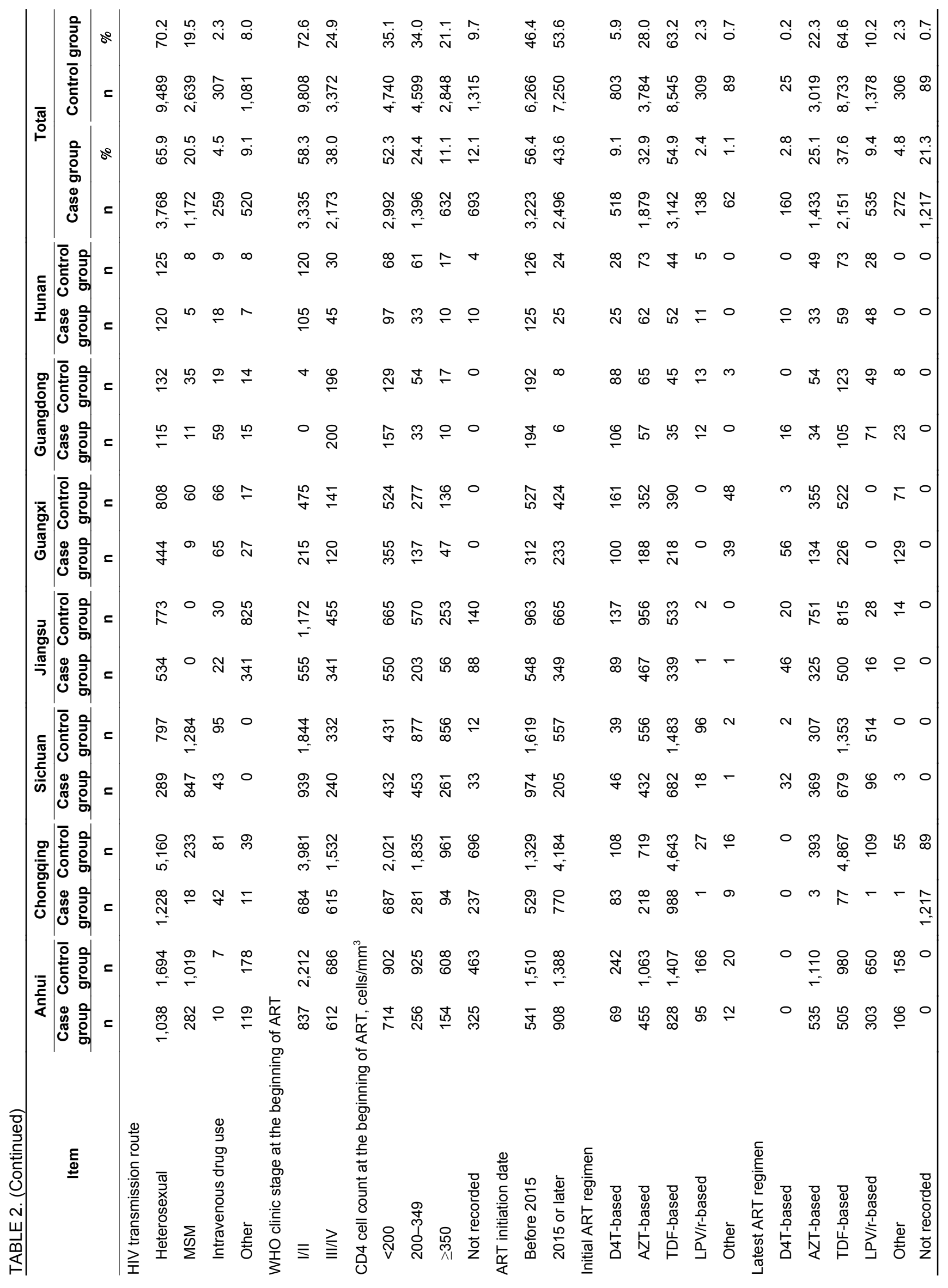




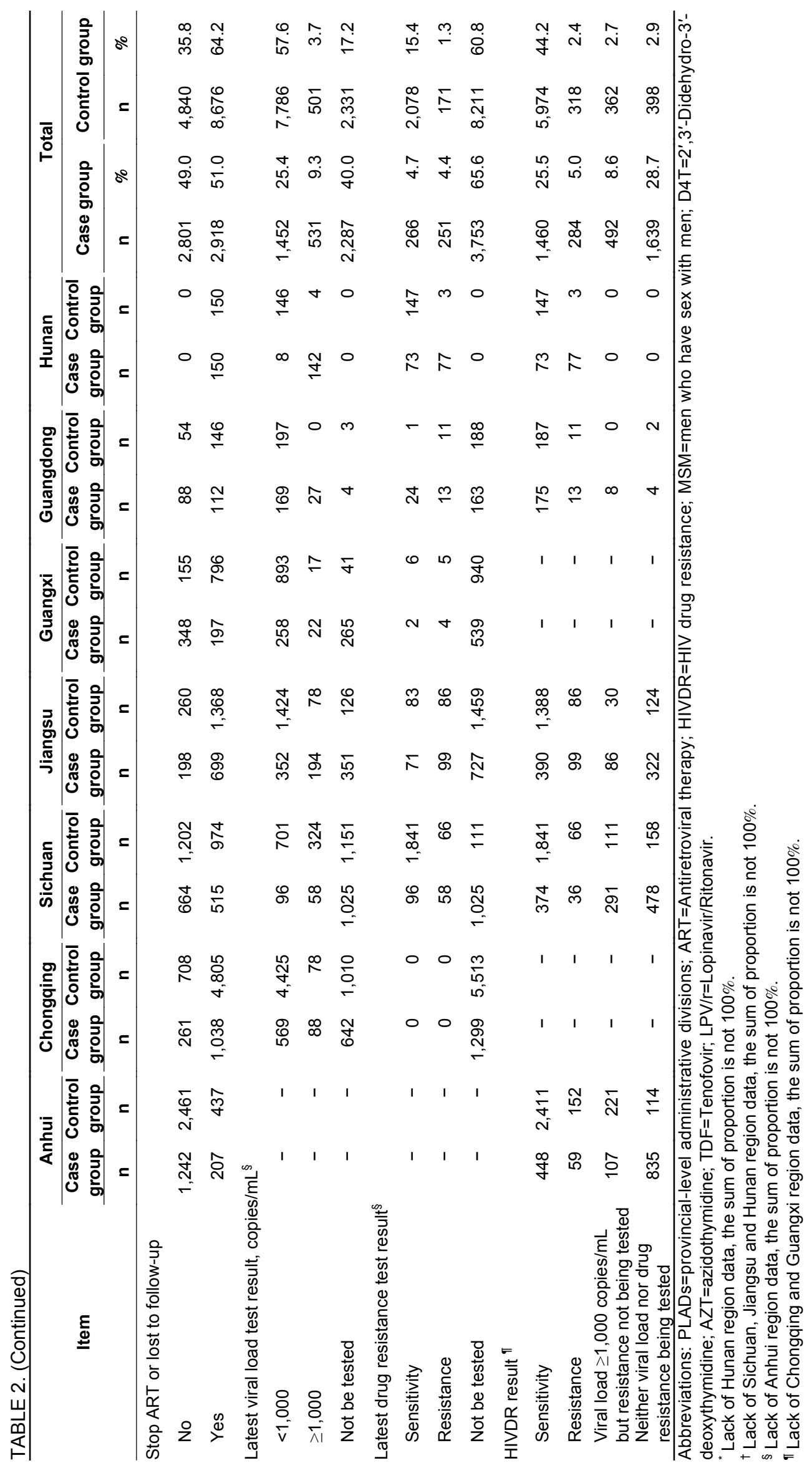


TABLE 3. Relationship between HIVDR and death in HIV-infected patients receiving ART.

\begin{tabular}{ll}
\hline \multicolumn{1}{c}{ HIVDR result } & AOR (95\% CI)* \\
\hline Sensitivity & 1 \\
Resistance & $4.25(2.10,8.62)$ \\
Viral load $\geq 1,000$ copies/mL but resistance not being tested & $4.65(1.74,12.39)$ \\
Neither viral load nor drug resistance being tested & $17.52(8.73,35.19)$ \\
\hline Abbreviations: ART=Antiretroviral therapy; HIVDR=HIV drug resistance; AOR=multivariate adjusted odds ratio; Cl=confidence interval. \\
* AOR was conducted by meta-analysis to merge the results of PLADs.
\end{tabular}

higher than that of the drug-resistant HIV-infected patients. Drug-resistant patients are prone to have poor adherence to ART treatment (5) and higher incidence of virological failure (G), which could accelerate deaths of HIV-infected patients. A previous study showed that the risk of mortality in drug-resistant patients was 3.26 times higher than that of the drug-sensitive population (95\% CI: 1.77-6.01), which was similar to the results of this study (7). This paper indicated that it is highly important to strengthen the drug resistance monitoring and prevention in HIV-infected patients receiving ART.

In accordance to the requirements of "Manual of the National Free Antiretroviral Treatment" (8), all treated HIV-infected patients with viral load $\geq 1,000$ copies $/ \mathrm{mL}$ should get drug resistance tested. However, the proportions of treated HIV patients with viral load $\geq 1,000$ copies $/ \mathrm{mL}$ but with drug resistance untested in some regions remained high in this study. Compared with drug-sensitive HIV-infected patients receiving ART, the risk of death was 3.65 times higher in patients with latest viral load $\geq 1,000$ copies $/ \mathrm{mL}$ but drug resistance untested. This result suggested that for those with viral load $\geq 1,000$ copies $/ \mathrm{mL}$, drug resistance tests should be routinely conducted to know patients' most updated drug resistance status. Therefore, ART regimens could be adjusted accordingly to improve treatment effectiveness.

Constant adherence is vital to effective ART for reducing viral load and HIV/AIDS-related opportunistic infections and mortality which used to be assessed by self-reported data of "missed doses in the past month" in previous studies (9-10). In this study, required viral load testing was used as a proxy for treatment compliance as it could promptly detect virological failure and drug resistance. The proportion of patients with latest viral load untested in the case group was higher than that in control group $(40.0 \%$ vs. 17.2\%). Compared with drug-sensitive HIV-infected patients receiving ART, the risk of death was 17.52 times higher than that of HIV-infected patients with neither viral load nor drug resistance being tested (95\%
CI: 8.73-35.19). This result indicates that during the ART follow-up management period, more attention should be paid on improving the adherence of patients, strengthening the follow-up quality of HIV clinics, and performing viral load and drug resistance testing promptly as required.

This study was subject to some limitations. First, the case-control design limited the ability to make causal inference about the proposed association. Second, some information of participants was not collected in some regions. Lack of these data may partly affect the analysis for the corresponding regions.

In summary, associations between drug resistance and death in HIV-infected patients receiving ART are highly related. It is important to strengthen the drug resistance monitoring and prevention in those patients. When conducting follow-up management of HIVinfected patients, adherence to antiviral treatment should be improved and viral load testing should be carried out as required. All treated HIV-infected patients with viral load $\geq 1,000$ copies $/ \mathrm{mL}$ should get drug resistance tested in a timely manner.

Acknowledgement: Anhui CDC, Chongqing CDC, Sichuan CDC, Jiangsu CDC, Guangxi CDC, Guangzhou Eighth People's Hospital, and Hunan CDC.

Conflicts of interest: The authors who have taken part in this study declared that they did not have any other potential conflicts of interest.

Funding: The Ministry of Science and Technology of China (2017ZX10201101, 2018ZX10721102-006) and National Natural Science Foundation of China (11971479).

\footnotetext{
doi: $10.46234 / \mathrm{ccdcw} 2021.068$

\# Corresponding authors: Yuhua Ruan, ruanyuhua92@163.com; Hui Xing, xingh09@163.com.

${ }^{1}$ State Key Laboratory of Infectious Disease Prevention and Control (SKLID), National Center for AIDS/STD Control and Prevention (NCAIDS), Chinese Center for Disease Control and Prevention (China CDC), Collaborative Innovation Center for Diagnosis and Treatment of Infectious Diseases, Beijing, China.
}

Submitted: December 16, 2020; Accepted: March 10, 2021 


\section{REFERENCES}

1. Zhang FJ, Pan J, Yu L, Wen Y, Zhao Y. Current progress of China's free ART program. Cell Res 2005;15(11 - 12):877-82. http://dx.doi.org/10.1038/sj.cr.7290362.

2. Dou ZH, Zhang FJ, Zhao Y, Jin CR, Zhao DC, Gan XM, et al. Progress on China's national free antiretroviral therapy strategy in 20022014. Chin J Epidemiol 2015;36(12):1345 - 50. http://dx.doi.org/10. 3760/cma.j.issn.0254-6450.2015.12.005. (In Chinese).

3. Zhao Y, Han MJ, Ma Y, Li DM. Preplanned Studies: progress towards the 90-90-90 targets for controlling HIV-China, 2018. China CDC Wkly 2019;1(1):5 - 7. http://dx.doi.org/10.46234/ccdcw2019.003.

4. Zuo LL, Liu K, Liu HL, Hu YH, Zhang ZJ, Qin JR, et al. Trend of HIV-1 drug resistance in China: a systematic review and meta-analysis of data accumulated over 17 years (2001-2017). EClinicalMedicine 2020;18:100238. http://dx.doi.org/10.1016/j.eclinm.2019.100238.

5. Bayu B, Tariku A, Bulti AB, Habitu YA, Derso T, Teshome DF. Determinants of virological failure among patients on highly active antiretroviral therapy in University of Gondar Referral Hospital, Northwest Ethiopia: a case-control study. HIV/AIDS Res Palliat Care 2017;9:153 - 9. http://dx.doi.org/10.2147/HIV.S139516.
6. Zuo ZB, Liang S, Sun XG, Bussell S, Yan J, Kan W, et al. Drug resistance and virological failure among HIV-infected patients after a decade of antiretroviral treatment expansion in eight provinces of China. PLoS One 2016;11(12):e0166661. http://dx.doi.org/10.1371/ journal.pone.0166661.

7. Zhang JH, Li HL, Shi HB, Jiang HB, Hong H, Dong HJ. Survival analysis of HIV/AIDS patients with access to highly antiretroviral therapy in Ningbo during 2004-2015. Chin J Epidemiol 2016;37 (9):1262 - 7. http://dx.doi.org/10.3760/cma.j.issn.0254-6450.2016. 09.016. (In Chinese).

8. Manual of the national free antiretroviral treatment. 4th ed. Beijing: People's Medical Publishing House.

9. Kan W, Teng T, Liang SJ, Ma YL, Tang H, Zuohela T, et al. Predictors of HIV virological failure and drug resistance in Chinese patients after 48 months of antiretroviral treatment, 2008-2012: a prospective cohort study. BMJ Open 2017;7(9):e016012. http://dx.doi.org/10.1136/bmjo pen-2017-016012.

10. Liu PT, Liao LJ, Xu W, Yan J, Zuo ZB, Leng XB, et al. Adherence, virological outcome, and drug resistance in Chinese HIV patients receiving first-line antiretroviral therapy from 2011 to 2015. Medicine (Baltimore) 2018;97(50):e13555. http://dx.doi.org/10.1097/MD.0000 000000013555 . 